\title{
A Case Study and Literature Review: Adenocarcinoma of the Lung
}

\author{
Piyush Vyas ${ }^{1}$, Marta Dudek ${ }^{1}$, Tomasz Głazewski ${ }^{1}$, Natalia Wąsowska ${ }^{1}$ and Seema Vyas ${ }^{2}$ \\ ${ }^{1}$ Medical University of Warsaw, Poland \\ ${ }^{2}$ Maria Sklodowska Curie memorial Centre and Institute of Oncology, Poland
}

Submission: March 0 4, 2017; Published: March 22, 2017

"Correspondence Address: Dr. Piyush Vyas M.D., Department of Oncology, Hematology and Internal Medicine ; Medical University of Warsaw Hospital, ulica Banacha 1a, Warsaw, Poland, Email: vyas_piyush20@yahoo.co.in

\begin{abstract}
We report case of a 48-year-old egyptian male patient with advanced lung cancer, who has been living for almost 9 years since the diagnosis. This is probably the first published case report of a patient with such long survival despite advanced disease. Current 5-year survival rate for this stage of lung cancers is 1-2\%. Still, there are few treatment options to use. Our patient, diagnosed in 2007 (with adenocarcinoma in the right lung), was qualified to chemotherapy, because the tumor was inoperable. According to the prevalent at that time chemotherapy standards he received first and second line of treatment: cisplatin with gemcitabine and then docetaxel, respectively. After having had been detected next progression of the disease (after second line treatment, he was qualified for targeted therapy with erlotinib: a tyrosine kinase inhibitor (TKI), namely the inhibitor of epidermal growth factor receptor (EGFR). During this third-line regimen we achieved long-lasting stabilization of disease with minimal side effects. At the time of publishing this manuscript he is undergoing 73rd cycle of erlotinib, while living high-quality life.
\end{abstract}

Keywords: Lung Cancer; Adenocarcinoma; Erlotinib; Third-Line Treatment; Tyrosine Kinase Inhibitor; Non Small Cell Lung Cancer; Liquid Biopsy

\section{Introduction}

Lung cancer is one of the most frequent malignancy in the world. It is the most common solid tumor type, after breast cancer in woman and prostate cancer in men. It is noticeable that every year as many people die from prostate, breast and colon cancers together as from lung cancer. It remains the most common cause of cancer-related death in men and the second in women. Incidence and mortality rates are still high each year there are reported 1.8 million of new cases $(13 \%$ of all new cancers diagnoses) and 1.6 million deaths $(20 \%$ of all cancer-related deaths)[1]. Tobacco - the most important risk factor - caused $71 \%$ of lung cancer deaths (almost 1 million in 2008). Now the incidence is beginning to decline, due to reduced smoking prevalence. Despite continuous advances in oncology the prognosis remains poor. The five-year survival rate for this cancer is averagely $10-15 \%$ [2] and when it is recognized in stage IV then only 1-2\%. Even early detected cancer in stage IA has only a $50 \%$ rate of five-year survival. The $80 \%$ of new diagnoses are connected with the metastatic diseases, and more than $50 \%$ are related with distant metastases. Median age at diagnosis is 71 years.

Based on therapeutic options, two broad types of lung cancer are distinguished: Non-Small Cell Lung (NSCL; approx.
15\%) and Small-Cell Lung (SCL) cancer. There are three many subtypes of NSCL cancer: adenocarcinoma, squamous-cell and large-cell carcinoma. The most common histological subtype of lung cancer is adenocarcinoma, which represents $40 \%$ of all lung cancers. Adenocarcinoma is also the most represented histotype of lung cancer in no-smokers. Non-smoking risk factors for lung cancer include diet, air polution, radon exposure and genetic susceptibility to cancer. Recently many mutations, which are present in different histological types of a tumor, have been discovered. Mutations in EGFR gene are common in Non-Small Cell Lung carcinomas. This gene encodes a transmembrane tyrosine kinase, comprising an extracellular ligand-binding domain and an intracellular domain with tyrosine kinase activity which transmits the signals via routes PI3K / AKT / mTOR RAS / RAF / MAPK and JAK / STAT. It regulates the many oncogenic functions - proliferation, survival, differentiation, angiogenesis, invasion and metastasis from local to distant. Activating mutation causes the initiation, promotion and progression of cancer. Symptoms that differ depending on the localization of the tumor are: cough, dyspnoea, haemoptysis, wheezing, chest pain, stridor, hoarseness, dysphagia, Pancoast sydrome, superior vena cava syndrome. In case of distant metastases symptoms 


\section{Cancer Therapy \& Oncology International Journal}

include also: weight loss, fatiguse, malaise and loss of appetite. Patients clinical presentation often compel doctors to work in multidisciplinary teams during the treatment process.

Stage II and III of NSCLC patients are good candidated for adjuvant chemotherapy. The CALGB study compared four cycles of paclitaxel-carboplatin in patients with resected stage IB NSCLC with significant benefit for tumors $>4 \mathrm{~cm}$. In stage IIIB and IV of NSCLC, cisplatin-pemetrexed regimen provided the same efficacy that cisplatin-gemcitabine. ALK-positive NSCLC patients, who received one prior platinum-based regimen or crizotinib, can be treated with ALK-targeting TKIs. New drugs such as antiangiogenic agents display improved efficacy in some patients subgroups. The introduction of targeted therapies has become an opportunity for patients with tumors resistant to traditional chemotherapy. One of such drug is erlotinib, which is an oral inhibitor of tyrosine kinase receptor, which acts on the epidremal growth factor receptor EGFR. The substance has low molecular weight that blocks a signal transduction and autophosphorylation. It has good penetration into the tumor tissue (63\%). It is administered orally and absorbed from the gastrointestinal tract in 59\%. Maximum serum concentration is achieved 4 hours after oral administration; binding to plasma proteins reaches 93\% [3]. Despite the good pharmacokinetics, the median survival observed in large clinical trials was 7.9 months with an average of 3.25 months without disease progression and percentage of annual survival ratio of $37.7 \%$ [4]. Currently erlotinib is used as a first or second line treatment of NCSL cancer, approved for EGFR mutations carriers.

The mutations, present in $20 \%$ of NSCL cancers, should be confirmed by testing the tumor tissue. However, Liquid Biopsy Test (Cobas EGFR Mutation test) remains the alternative way to identify patients with NSCL cancer qualified for EGFR-inhibitors. The diagnosis can be established by testing the blood or urine for specific mutations in EGFR gene. This is non-invasive procedure based on analysis of isolated DNA. Liquid biopsy can be considered, when the standard biopsy from tumor tissue is impossible to conduct. Solid research on its efficacy should be undertaken anyway. It seems that in the near future easier and faster lung cancer diagnosis will be more accessible for patients excluded from standard histological examination.

\section{Case Study}

In 2007, 48-year-old Egyptian patient, with no chronic internal diseases, presented to the Clinical Hospital in Warsaw with superior vena cava syndrome. He was initially placed in the Department of Hypertension and Internal Diseases. During preliminary diagnostic procedures suspicious tissue growth was detected in the cavity of the right lung- from histopathology report it was adenocarcinoma, CS IV. The patient was transferred to the Department of Oncology. There was no possibility to surgical treatment. The decision was made to use the first line of chemotherapy - combination of cisplatin with gemcitabine.
Under the careful observation, the tolerance of treatment was satisfactory. However, the progression of the disease (increasing tumor size) forced us to use the second line of the chemotherapy - docetaxel. Despite the change of treatment the progression was proceeding. Next, without confirmation of EGFR mutation, we decided to introduce erlotinib at a dose of $150 \mathrm{mg}$ per day orally (it has been shown to be effective in patients with or without EGFR mutations, but appears to be more effective in patients with EGFR mutations).

At the time we administered first cycle of treatment with targeted drug the confirmation of the mutation presence was not required officially (it was a non-standard procedure). Moreover, further treatment was continued on the basis of a positive therapeutic response - we obtained stabilization of disease. Unresectable nature of the disease indisposed standard biopsy because of the high risk of adverse events during such surgery. Simultaneously, the liquid biopsy was unavailable at this time. After the ineffective chemotherapy concurrently the patient received palliative radiotherapy. At the time of taking erlotinib lumpy change was in the bay right lung in the area of the upper lobe bronchus and had dimension $22 \times 18 \mathrm{~mm}$, with the necro-fibrous changes in the central area of the right lung. The patient tolerated targeted therapy well, did not report any serious side effects apart from a small papular rash on the skin of the back and trunk. His general condition was very good (WHO 0). Frequent control tests (CT scans with contrast every 3 months) was showing continuingly stable disease. The dimensions of described mass did not change, remaining $23 \times 18$ $\mathrm{mm}$. Additionally, scans did not revealed metastatic disease. In the initial period of therapy the patient was not taking any other medications aside from erlotinib [5-7].

In 2013, the patient was diagnosed with type II diabetes and started treatment with oral hypoglycemic drugs. In 2014 the patient was hospitalized due to the bleeding from the lower gastrointesinal tract with hemorrhoids detected together with multiple polyps of the colon and the sigmoid colon diverticulum. In subsequent $\mathrm{CT}$, there was still no progression of cancer. We performed also PET-CT scan in March 2015 during the 60th cycle of erlotinib, which showed increased FDG uptake (SUV max $2.5)$ in cancerous change in the lung. Due to a good tolerance of the treatment and persisting stabilisation, the patient had been treated with 73rd cykle of erlotinib, and still lives in good general condition - WHO 1.

\section{Discussion}

There are several targeted therapy options for EGFR, ALK, ROS1 positive non small cell lung cancer patients. For example for EGFR mutated NSCLC patients (mutated in 30\% to $40 \%$ of Asians and in 10-20\% of Caucasians) the options include Erlotinib (60\% - 80\% predicted response rate) Gefitinib (70\% predicted response rate), Afatinib (60\% predicted response rate), Osimertinib (50\% - 60\% predicted response rate where $\mathrm{T}$ 
$790 \mathrm{M}$ mutation is present), Almost $1 \%-7 \%$ adenocarcinomaof lung have ALK mutation, where ALK inhibitors (HSP 90 inhibitors like Crizotinib, Ceritinib or Alectinib can be potential options. About 1,7\% patients with adenocarcinoma would have ROS1 oncogene mutations, and among these patients Crizotinib can be a potential therapeutic option. Other therapeutic options are dacomitinib, a therapeutic agent (15 predicted response rate) of HER2 oncogene is mutated, the mutation prevalence only $2 \%$. Therapies targeting oncogenes RET (therapeutic agent RET inhibitor Cabozantinib), oncogene BRAF (targeted agent BRAF/ mek inhibitors like Dabrafenib, Tramantinib), oncogene MET (therapeutic target -Crizotinib), NTRK (therapeutic target -Crizotinib/NTRK inhibitors) are some of the potential future options.

A special reference should be made about EGFR mutations .Del 19 and L858R constitute to about 90\% of mutation in EGFR (Exon 18 substitution 5\%; Exon 19 deletion -45\%, Exon20 insertions <1\%; Exon 21 point mutations 40-45\%).

Worth remembering are the two pathbreaking trials IPASS and EURTAC trials. In IPASS trial, the PFS of Gefitinib arm was 9.5 months versus 6.3 months in the chemotherapy arm. The chemotherapy used to treat patients in this trial was carboplatin plus paclitaxel. In EURTAC trial Erlotinib it was shown that PFS in Erlotinib arm was 10.4 month versus 5.2\% months [8].

Afatinib consideredII-generation EGFR-TKI is an irreversible TKI which targets EGFR, HER2 and HER4 oncogenes. Worth mentioning are the results of LVX-Lung 3 and LUX-Lung6 where primary endpoint was also PFS. LUX Lung 3 showed a PFS of 13.6 months versus 16.9 months (for chemotherapy) LUX-Lung6 showed PFS of 11 months (Afatinib ) vs 5-6 months (chemotherapy Cis/Gem). Worth an impressive OS of 33 months (afatinib) vs 21.1 months (chemotherapy) was shown. In luxlung 6 the median OS was 31.4 months vs 18.4 months (afatinib vs chemotherapy Cis/Gem) [9]. Further important are the results of the latest LUX- Lung 7 trial where Paz-Aves L et.al. showed median PFS of 11.0 months vs 10.9 months (Afatinib vs Gefitinib arm); median TTF 13.7 vs 11.5 months (Afatinib vs Gefitinib ) and ORR 10.1 vs 8.3 months (DoR-duration of response) in afatinib vs Gefitinib arm [10].

Interestingly the OS shown is LVX-Lung 7 was 27.9(Afatinib arm) vs 24.5 (Gefitinib arm). EGFR mutated patient with Del 19 had median overall survival of 30.7 (afatinib) vs 26.4 months (Gefitinib). Among L858R EGFR mutated patients OS was 25.0 vs 21.2 months.LUX- Lung 8 showed PFS 2.6 vs 1.9 months (Afatinib vs Erlotinib) and OS 7.9 vs 6.8 months (Afatinib vs Erlotinib).

Other trials worth mentioning in this article which may shape future therapies in adenocarcinomas of lung or patients with EGFR oncogene mutations are J025567, a phase 2 study comparing Erlotinib 150mh/day with Bevacizumab 15mg/ kg q3w vs Erlotinib. It was shown that PFS in Erlotinib with Bevacizumab arm was 16 months vs 9.7 months in Erlotinib arm [11], Beverly trial, a phase III study is now planned which would also compare Erlotinib 150mg vs Erlotinib 150mg. with Bevacizumab $15 \mathrm{mg} / \mathrm{kg}$ iv every 1 days, RELAY trial which started in May 015 is presently com paring ramucirumab 10mg/ $\mathrm{kg}$ every weeks and Erlotinib 150mg. daily vs placebo and Erlotinib 150mg/ day. Primary endpoint of this trial is PFS [12]. Yang et. al. during European Lung cancer conference April 2016 showed potential activity of Osimartinib in second line. Acquired T790M+ patients where PFS of 11 months was attained. AURA 3 study, a phase III, open label, randomized study is now planned which would compare Osimartinib (80mg. p.o.q.d.) with patients receiving platinum based doublet chemotherapy every 3 weeks among patients receiving second line therapy. (NSCLC EGFR M+ progressor on EGFR TKI). Awaited are results of FLAURA trial in ASCO 017 probably which compared Exon 19 del IL858R EGFR mutations among Asians and non-Asian patients comparing Osimartinib (80mg. p.o.q.d.) vs EGFR-TKI, standard of care Gefitinib or Erlotinib.

Thus it is worth concluding that EGFR molecular testing is essential for every adenocarcinoma. Till date standard of care among EGFR mutated patients are Erlotinib, Gefitinib and Afatinib. As far as Afatinib is concerned, it has shown overall benefit in EGFR exon 19 mutated lung cancers, it has proved to be active in 'uncommon mutations" for eg. In LL7 mutation. It appears that Erlotinib and bevacizumab can be active, results of trails are pending.

\section{Conclusion}

Our patient, who was diagnosed with adenocarcinoma of the right lung stage IV, has been effectively treated with erlotinib in monotherapy for almost 6 years. To the best of our knowledge, this is probably one of the longest surviving patient with Non-Small-Cell Lung cancer [5,6]. After the failure of initial chemotherapy regimens (cisplatin with gemcitabine and then docetaxel), it was possible to start third-line effective treatment with targeted therapy - erlotinib. Until now, this patient tolerates therapy very well. Erlotinib is well-established EGFR inhibitor for the treatment of nonoperablelocally advanced or metastatic Non-Small Cell Lung cancer in those who carry EGFR gene mutations [7]. Overall response rate are about $50 \%$ better than standard chemotherapy. Thus we have shown if properly treated life of advanced adenocarcinoma patients can be prolonged significantly. Now in the era of targeted therapies, it seems that future has a lot to offer. A practicing oncologist must have a good knowledge about the targeted therapies so that he can select the optimum therapy, and prolong life of his patients, especially in the field of treatment of advanced adenocarcinoma of the lung.

\section{Reference}

1. WHO (2017) World Cancer Report 2014, Geneva, Switzerland.

2. Cooper WA, Lam DC, O’Toole SA, Minna JD (2013) Molecular biology of lung cancer. J Thorac Dis 5(Suppl 5): S479-S490. 


\section{Cancer Therapy \& Oncology International Journal}

3. J Smith Erlotinib (2005) Small-Molecule Targeted Therapy in the Treatment of Non-Small-Cell Lung Cancer. ClinTher. 2005 Oct;27(10):1513-34

4. Reck M, van Zandwijk N, Gridelli C, Baliko Z, Rischin D, et al. 2010) Erlotinib in Advanced Non-small Cell Lung Cancer Efficacy and Safety Findings of the Global Phase IV Tarceva Lung Cancer Survival Treatment Study J Thorac Oncol 5(10): 1616-1622.

5. Polychronidou G, Papakotoulas P (2013) Long-Term Treatment with Erlotinib for EGFR Wild-Type Non-Small Cell Lung Cancer: A Case Report. Case Rep Oncol 6(1): 189-196.

6. Rocha-Lima CM, Raez LE (2009) Erlotinib (Tarceva) for the Treatment of Non-Small-Cell Lung Cancer and Pancreatic Cancer 34(10): 554$556,559-564$.

7. Kobayashi K, Hagiwara K (2013) Epidermal growth factor receptor (EGFR) mutation and personalized therapy in advanced nonsmall cell lung cancer (NSCLC). Target Oncol 8(1): 27-33.

8. Khozin S, Blumenthal GM, Jiang X, He K, Boyd K, et al. (2014) U.S. Food and Drug Administration approval summary: Erlotinib for the firstline treatment of metastatic non-small cell lung cancer with epidermal growth factor receptor exon 19 deletions or exon 21 (L858R) substitution mutations. Oncologist 19(7): 774-779.

9. Yang JC, Wu YL, Schuler M, Sebastian M, Popat S (2015) Afatinib versus cisplatin-based chemotherapy for EGFR mutation-positive lung adenocarcinoma (LUX-Lung 3 and LUX-Lung 6): analysis of overall survival data from two randomised, phase 3 trials. Lancet Oncol 16(2): 141-151.

10. L. Paz-Ares, E.H. Tan, L. Zhang, V. Hirsh, K. O’Byrne (2016) Afatinib (A) vs gefitinib (G) in patients (pts) with EGFR mutation-positive (EGFRm+) non-small-cell lung cancer (NSCLC): overall survival (OS) data from the phase IIb trial LUX-Lung 7 (LL7). Ann Oncol 27(suppl_6): LBA43.

11. Sets T (2014) Erlotinib Alone or With Bevacizumab as First-Line Therapy in Patients With Advanced Non-Squamous Non-Small-Cell Lung Cancer Harbouring EGFR Mutations (J025567): An Open-Label, Randomised, Multicentre, Phase 2 Study. Lancet Oncol 15(11): 12361244.

12.S Ramalingam, JC H Yang, CK Lee, T Kurata, D W Kim, et al. (2016) Deferred publication: Advanced NSCLC (Abstract LBA2_PR) 11 Suppl 4S: S152-S155.

\section{Your next submission with Juniper Publishers} will reach you the below assets

- Quality Editorial service

- Swift Peer Review

- Reprints availability

- E-prints Service

- Manuscript Podcast for convenient understanding

- Global attainment for your research

- Manuscript accessibility in different formats

( Pdf, E-pub, Full Text, Audio)

- Unceasing customer service

Track the below URL for one-step submission https://juniperpublishers.com/online-submission.php 